
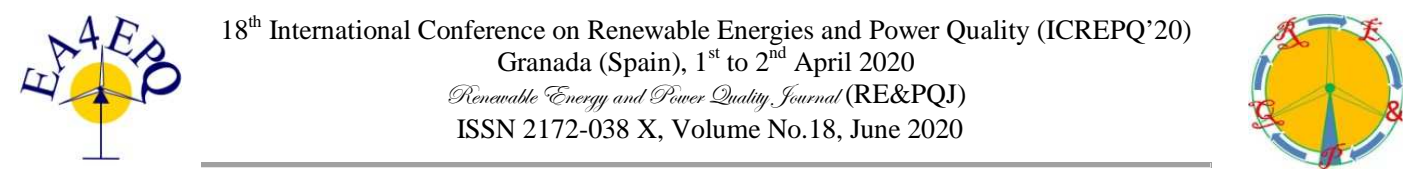

\title{
Importance of Parameterization to Improve Meta-heuristics Performance for Smart Grid Applications
}

\author{
G. Juarez ${ }^{1}$, O. Abarrategi ${ }^{1}$, P. Eguia ${ }^{1}$ \\ ${ }^{1}$ Department of Electrical Engineering \\ Escuela de Ingeniería de Bilbao, UPV/EHU \\ Rafael Moreno Pitxitxi, 48013 Bilbao (Spain) \\ Phone/Fax number:+0034 6014971, e-mail: oihane.abarrategui@ehu.eus
}

\begin{abstract}
The development of the Smart Grid requires handling big amounts of data and solving large combinatory problems under uncertainty. Meta-heuristic algorithms are a useful tool to handle such problems.

One of the main problems of these algorithms is that they have to operate in real time. Thus, it is necessary to reduce computation times as much as possible. This paper demonstrates that an extensive empirical study of parameterization leads to a very significant improvement of algorithm efficiency. It focuses on Item Oriented Ant Colony Optimization (IOACO) algorithm reducing by one third the computation time of the most efficient method in the references.
\end{abstract}

Key words. Meta-heuristics, Parameterization, Smart Grid, Optimization, Distributed Generation

\section{Introduction}

Currently, the Electric Power System is transitioning from the traditional vertical system to an integrated Smart Grid that will allow a bidirectional information and power flow. The Smart Grid has to be a decentralized, autonomous system that will have to deal in real time with large amounts of data and plenty of combinatory problems under uncertainty. In such a system, the use of meta-heuristic algorithms can prove very useful.

Plenty of meta-heuristic based tools have been used to solve problems in the electric power system during recent years. Thus, Flower Pollination Optimization was proposed for optimal sizing and location of capacitor banks. The objective function was designed to reduce the total cost [1]. [2] proposed Tabu Search (TS) for Transmission network planning. [3] used a hybrid approach of both Genetic Algorithms and fuzzy logic for hydroelectric generation scheduling. Particle Swarm Optimization (PSO) was used for short term Load forecasting [4]. Ant Colony Optimization (ACO) was proposed by [5] for service restoration in power distribution systems.
PSO has been used to solve the problem of optimal location of Distributed Generation (DG), as well as Genetic Algorithms [GA] [6-9]. PSO has also been proposed by [10] for optimal location and sizing of DG and Distribution STATCOM (DSTATCOM) with the aim of reducing the total power loss and improving voltage profiles in radial distribution systems.

A review by [11] of meta-heuristic methodologies applied in the area of power converters highlighted the importance of the optimization tools, and the many benefits they provide to tackle the challenges encountered in the design, operation, and control of power converters.

Another classical problem is Feeder Reconfiguration for loss reduction [12] for which different meta-heuristics have been used: Genetic Algorithms [13, Neural Networks [14], Simulated Annealing (SA)[15-16] and TS [17]. Ant Colony Optimization has also been used for loss minimization through feeder reconfiguration [18-19] and also in a Smart Grid approach to favour DG integration by [20-21].

Therefore, it is possible to see the importance of metaheuristics for combinatory optimization problem solving in the Power System. The importance of parameterization for this kind of algorithms is known, and although its determination is a small part of most studies the focus does not normally lie on it on most of the references. When improvements want to be made, new more refined methodologies are designed with different degrees of success. However, this papers proposes a more careful approach to parameterization in order not only to obtain satisfactory results but also reduce significantly the operation times and efficiency of previously used methodologies. For this study IOACO proposed by [21] as it was the most efficient than [18-20]. 59 experimental trials have been conducted in order to prove that computation times can be significantly reduced, whilst the good results of the algorithm are maintained. 


\section{Item Oriented Ant Colony Optimization}

This methodology was proposed by [6] and it proposes to minimize distribution grid losses and maximize DG penetration by using feeder reconfiguration [5][6]. For that purpose it uses a methodology based on Ant Colony optimization.

The optimization, in this case, is the reconfiguration of the distribution network. A normally open switch (tie switch) and a set of normally closed switches (sectionalizing switches) linking one substation to another form a loop.

To make the distribution network radial, a tie switch has to be selected in each loop. The appropriate tie switch combination minimizes the power losses.

Loops gathering groups of remote controlled switches of feeders between substations form the Search Space. Each loop is composed by the switches that form the vertices of the Search Space. Thus, the loops consist on a succession of switches, each of them identified by a number or code.

Unlike in previous feeder reconfiguration methodologies using ACO, the four methodologies proposed in this paper do not consider the feeder reconfiguration problem as an ordering problem. Thus, the pheromone and the function fitness are associated directly to the items, not to the path among items.

In the new methodology proposed (Item Oriented Ant Colony Optimization-IOACO), the number of items that form the solution is fixed. Also, at least one item from each loop has to be selected. However, it is not specifically an ordering problem, because the information is attached to the item and not to the path between items. It is the choice of the item itself that defines the solution fitness and not the path followed to construct this solution.

The power losses are calculated on an n-node system of known topology. The location and characteristic of the demand, as well as the generation, are known and constant. The base for the calculation of power losses is the power flow.

Also, the optimization problem has to comply with the network stability constraints. Bus voltages have to be kept within limits, the thermal rating cannot be exceeded and no islanded operation can happen.

A penalization factor Lambda is applied every time a constraint violation is detected, for each violated constraint. Thus, taking into account the penalization of violations, the objective function is defined by (1), whilst the constraints (2) for voltage and (3) for current.

$$
\begin{aligned}
& m i n F=\min \left(P_{\text {leaso }}+\lambda_{v} C_{v}+\lambda_{i} i C_{i}+\lambda_{2 M} C_{L M}\right) \\
& V_{\min } \leq\left|V_{i}\right| \leq V_{\max }
\end{aligned}
$$

$\left|I_{i}\right| \leq I_{i} \max$

Once this is done, the item fitness eta can be calculated. Whenever the objective function decreases, that is when losses are minimized whilst constraints are kept, the fitness value of the item increases, so the fitness is good when the losses are small.

$$
\eta(k, t)=\frac{1}{P_{\text {losgss }}+\lambda_{v} C_{v}+\lambda_{i} i C_{i}+\lambda_{L M} C_{L M}}
$$

Initially, a fixed pheromone amount is assigned to all items, so that all items have the same possibilities to be included in the search. Then the ants are placed randomly through the search space.

Afterwards the ants have to make a move. For example the ant in item 4 of loop 1 has to choose an item of loop2. For the transition the state transition rule is used, as shown in (5).

$$
P i=\left\{\begin{array}{cr}
\frac{[\tau(t)]^{\alpha}[\eta(k, t)]^{\beta}}{\sum_{\text {jeloop }}[\tau(t)]^{\alpha}[\eta(k, t)]^{\beta}} & \text { if jeloop } \\
0 & \text { otherwtse }
\end{array}\right.
$$

Where:

$\begin{array}{ll}\mathbf{k} & \text { ant } k \\ \mathbf{t} & \text { step that ant } k \text { takes } \\ \boldsymbol{\tau} & \text { pheromone deposited in a certain item } \\ \boldsymbol{\eta} & \text { fitness, pseudo-utility, the local heuristic } \\ & \text { associated to the item } \\ \boldsymbol{\alpha} & \text { weighting factor for the pheromone } \\ \boldsymbol{\beta} & \text { weighting factor for the heuristic }\end{array}$

The pheromone and fitness of each item is used to calculate the probability that the item has to be chosen. The probability is calculated taken into account the pheromone and the fitness of the item. To determine the importance of pheromone vs. fitness, weighting factors alpha and beta are used. When this is done a "virtual dice" is thrown and the ant makes the move.

Once an item of all loops have been chosen, the pheromone related to the items is updated applying the global updating rule.

$\tau_{i}=(1-\delta) \tau_{i}+\delta \Delta \tau_{i}$

$\Delta \tau_{i}=\frac{Q}{\eta_{\text {hest }}}$

Where:

$\tau_{\mathbf{i}}$ pheromone accumulated in item $\Delta \boldsymbol{\tau}$ pheromone variation 

-global updating rule\|

(1- $\boldsymbol{\delta})$ pheromone evaporation rate for the global updating rule

Q constant that depends on case features

$\boldsymbol{\eta}_{\text {best }}$ fitness of the best global ant

\section{Network model}

The distribution network is a simple three feeder system of $11.4 \mathrm{kV}$, as can be seen on Fig.1. Further network data can be found in [5-6]. It is a meshed network which is operated as a radial network. Besides, it has sixteen switches, three of which have to remain open (three tie switches) in order to make radial operation possible.

The total load power consumption amounts $28.7 \mathrm{MW}$ and 17.3MVAr and the installed capacitor banks provide 11.4MVAr.

This distribution network has been modeled in PSS/E 30.3.2 according to the data presented in [5-6]. The losses calculated for this model's original configuration are $0.5114 \mathrm{MW}$. The original configuration s formed by tie switches 15, 26 and 21.

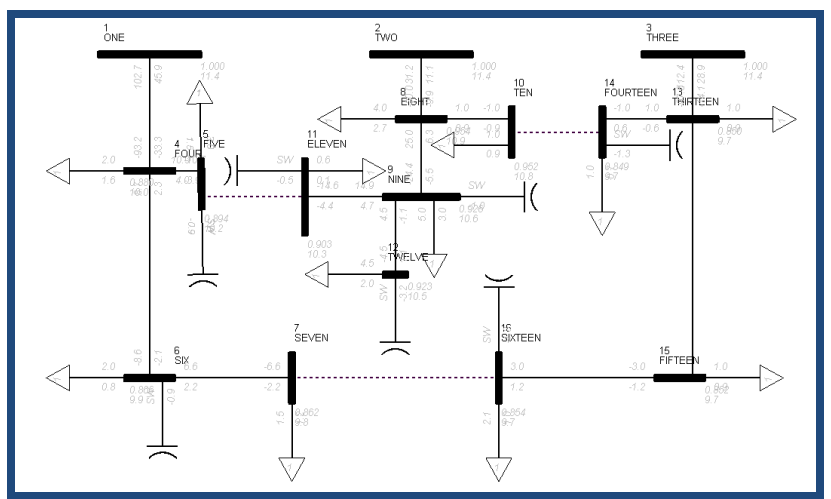

Fig. 1. :3 Feeder system

\section{Parameters}

The parameters we will change and analyze are the following:

- Ant number n: How many virtual ants take part in the solution construction each iteration

- Cycles/Iteration number Z: How many times will the search be repeated.

- $\lambda_{\mathbf{v}}, \lambda \mathbf{i}, \lambda_{\text {islanding: Penalizations in case there are }}$ network constraint violations. When voltage goes out of range, $\lambda_{\mathbf{V}}$ applies. If current exceeds the thermal limits of the line, $\lambda \mathbf{i}$ applies and if any network area experiences loss of mains, $\boldsymbol{\lambda}_{\text {islanding }}$ applies.
- $\quad \boldsymbol{\alpha}:$ is the weighting factor for pheromone, i.e., a constant used to weight the experience gathered from previous ant search. High values favour the importance of pheromone accumulation in the search.

- $\quad \boldsymbol{\beta}$ : weighting factor of the fitness, it favours the efficiency of a solution. High values favour exploration of new and valid solutions.

- $\boldsymbol{\delta}$ : pheromone evaporation rate. When the search finishes, it manages how pheromone is accumulated in the items, how quickly it accumulates and how quickly it evaporates.

- $\tau_{0}$ : initial pheromone level for all items.

- Q: constant that determines the velocity of pheromone accumulation

\section{Results}

A total of 59 trials have been done in the Three Feeder system. This paper presents some of the most significant study cases. For the sake of an easier understanding trials have been divided into seven big groups. The results of five of those are especially significant and of those five, the two most significant cases will be presented in this paper. The original configuration consists on switches [15,26,21] and the losses associated to it are $0.5114 \mathrm{MW}$. The optimal solution for this system $[26,19,21]$ reduces an $8,9 \%$ the losses to $0.4682 \mathrm{MW}$.

Table I. - Pheromone accumulation for higher lambda values

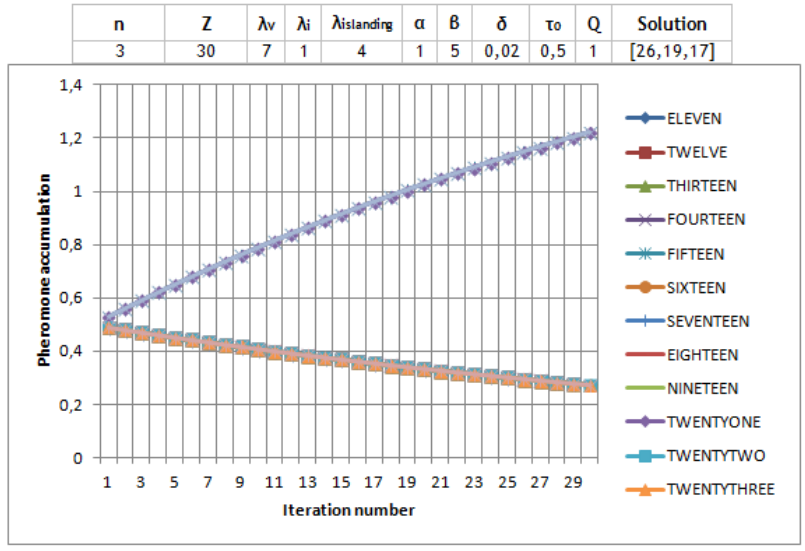

The first group consists on assigning different values to the penalization factors. Assigning different values or the same for all penalizations did not make a difference. However, there was a difference in the quality of the search when values of Lambda are lower. Although, in any case, the algorithm achieved the optimal solution, for high lambda values the search is stagnant and exploration is very little as can be seen in Table I, whereas for lower Lambda values the search is better and more explorative and less based on exploitation. 
Table II. - Pheromone accumulation for lower lambda values

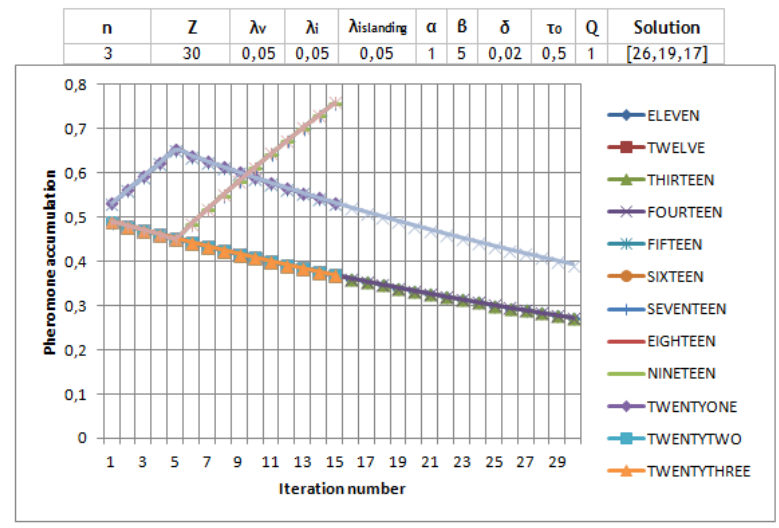

The second group intends to analyze the effects on changes to the weighting factors of pheromone and fitness, $\alpha$ and $\beta$ respectively. In this case, it is the relationship between the two weighting factor that matters. If $\alpha=\beta$, the solution found is not the optimal, resulting on higher losses, independently on the value we assign to those, as seen on Table III.

Table III. - Pheromone accumulation for $\alpha=\beta$

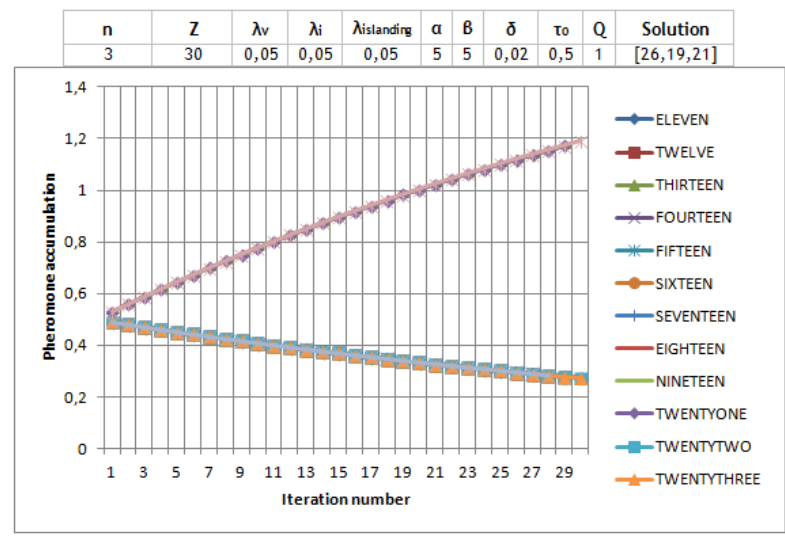

The same happens when $\alpha>\beta$, stagnation takes over as pheromone guides the search process to strongly. Thus, it is for $\beta>\alpha$, when exploration and fitness are favoured that the search is rich and the results are optimal, as seen in Table IV.

Table IV. - Pheromone accumulation for $\alpha>\beta$

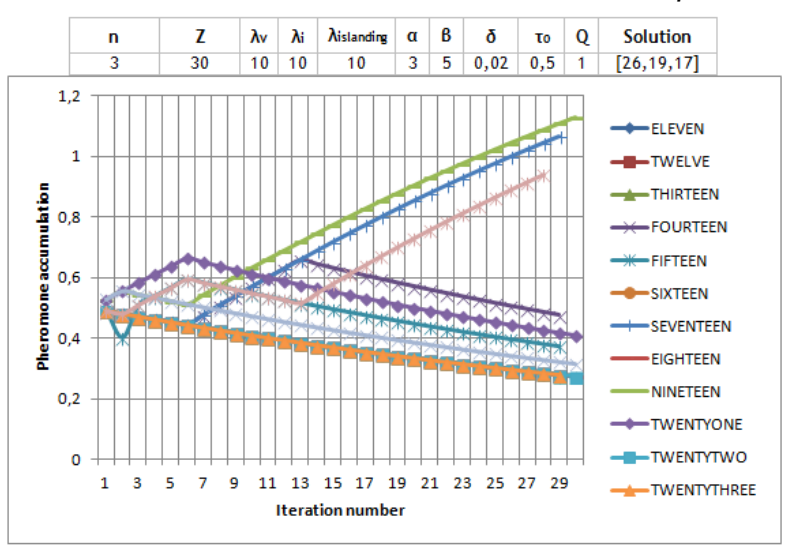

Next group analyzed relationships between $\delta$ and $Q$, the pheromone evaporation rate and velocity respectively. Q values around 10 and low $\delta$ values yield good results.

If pheromone evaporates too slowly, exploration leads the search and as we have seen, this tends to lead to suboptimal solutions or homogeneous and stagnant search processes. Table V shows a very reach and explorative processes when most part of the search space is taken into account. The optimal solution is obtained, with the correspondent loss reduction.

Table V. - Pheromone accumulation for $\delta<0.1$ and $\mathrm{Q}=8$

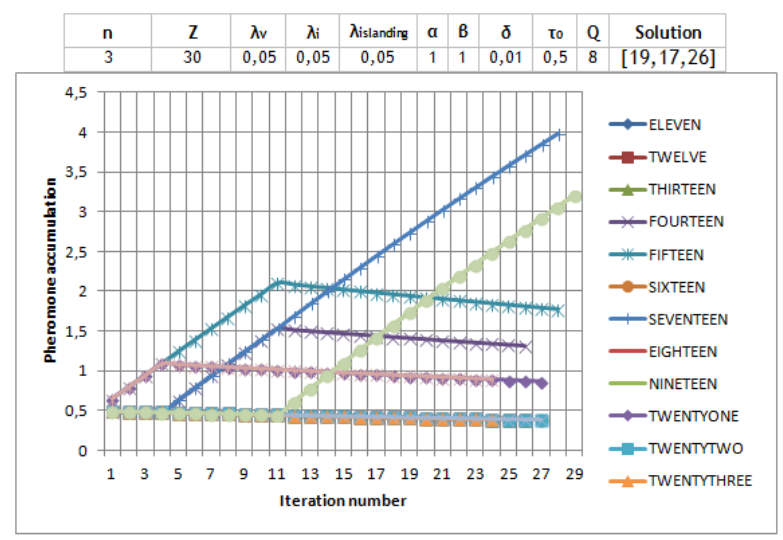

The number of ants constructing the solution in parallel each cycle has been analyzed. The trials showed that there was little benefit to reducing or increasing the ant number, as long as the other parameters maintained the right values. A too small ant number $(<3)$ is not recommended as it reduces the explorative potential of the algorithm, however, bigger values do not contribute to better solutions.

As seen in Table VII, the increase of the ant number does not lead per se to a good solution, in fact, in this case a suboptimal solution has been obtained and the search process is not especially good. Thus, it is not necessary to increase the ant number which would be counterproductive for the computation time and efficiency.

Table VII. - Ant number

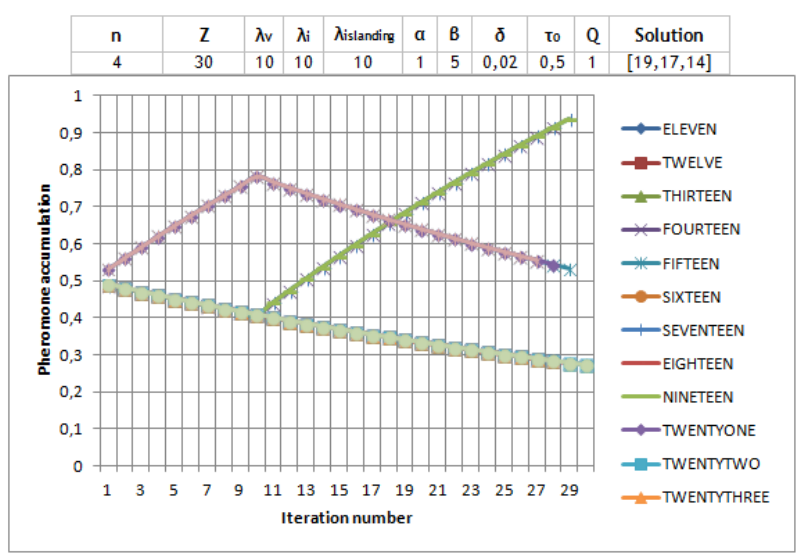


In order to decrease computation times, the next set of trials were intended to reduce the cycles or iteration numbers of the algorithm. If it is possible to obtain the optimal solution and conduct appropriate search processes with fewer cycles, computation time is spared and the efficiency of the algorithm increases.

Therefore, a set of trials was made in order to prove that the previously detected successful parameter relations led to a gain of efficiency in the algorithm.

As it is possible to observe in Table VI., keeping the original parameterization values of IOACO does not allow an iteration reduction. In this case, the algorithm was not able to find the optimal solution, the pheromone accumulated quickly in the same tie switches and the search process was stagnant.

Table VI. - Cycle number reduction, inadequate parameterization

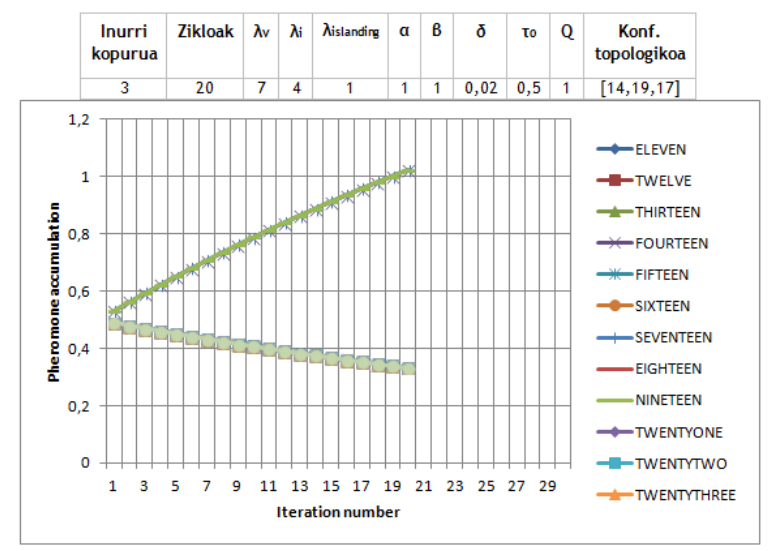

However, as it is possible to observe in Table VII when the other parameters are set at the appropriate values, as tested in the previous trials, it is possible to reduce the cycle number from 30 to 20 , i.e. one third, and still have very satisfactory results. Table VII shows a heterogeneous and rich search process and the optimal solution was obtained.

Table VII. - Cycle number reduction, adequate parameterization

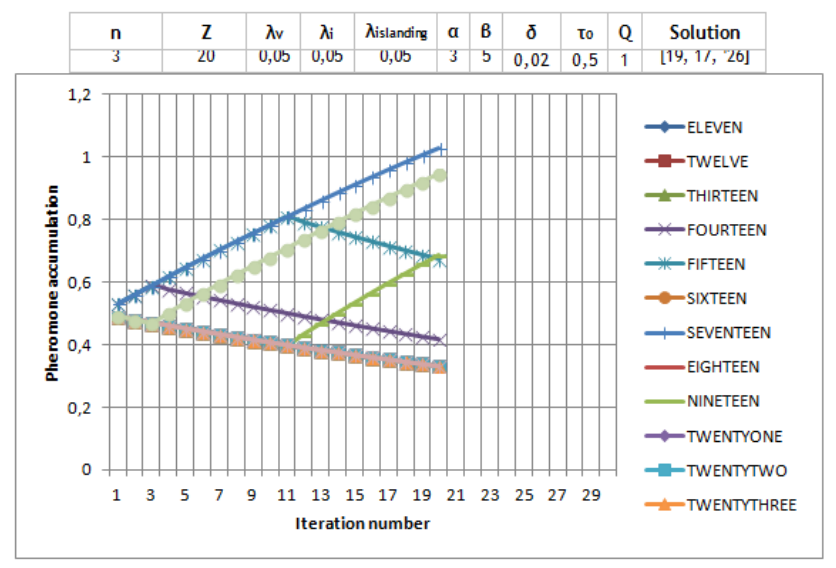

Finally, Table VIII keeps the recommendations for parameter relation but changes the value for the exploration and exploitation weighing factors $\alpha$ and $\beta$.
The results are satisfactory, showing that it is possible to obtain the optimal solution and reduce the cycle number thus improving the efficiency of the algorithm. Therefore, it confirms the fact that the parameter relation and not the exact value is what lead to successful solution construction.

Table VIII. - Cycle number reduction

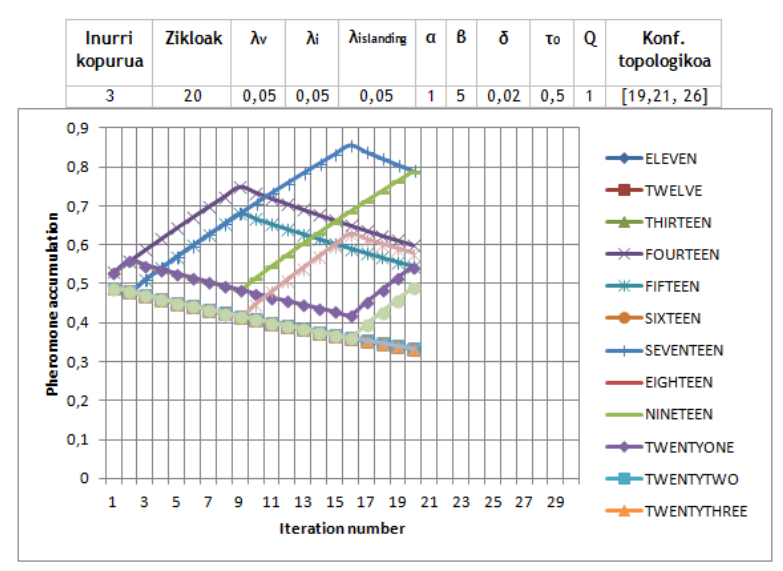

\section{Conclusion}

The experimental parameterization trials led to an efficiency improvement of a $33.33 \%$ by reducing computation time of the most efficient methodology in the literature [21] in one third.

Parameterization strongly influences de search ability of the algorithm. The search is more efficient and thorough depending not only in the values of the parameters but also on the relation among them.

Thus, the parameterization guidelines obtained from the study are relational which means that its conclusions can be used for different distribution systems, apart from the one considered in the study cases.

The parameterization guidelines obtained from the study are summarized hereafter.

When using smaller Lambda $\lambda$ the search process improves significantly. Using high Lambda values results in a fast pheromone accumulation and the stagnation of the search process.

When the values of $\alpha$ and $\beta$-are equal, regardless of their values, problems appear on the search process. However, if small Lambda values maintained, the results can be satisfactory. Nevertheless, the best results and more heterogeneous and rich search processes can be obtained by maintaining a $\mathrm{B}>\alpha$ proportion

Another factor that contributes to efficient search processes and good results is the combination of low $\delta$ values and high $\mathrm{Q}$ values. 
If there are big value differences between parameters, or extreme parameter values are used, search processes are poor and the solutions obtained present high loss values.

Increasing the number of ants that simultaneously build a solution does not significantly affect the results, and leads to higher computation times.

Decreasing the number of cycles, whilst following the guidelines presented for the other parameters leads to very good results.

Thus, it can be said that by using the right parameterization it is possible to reduce the cycles of the algorithm and reduce computation times significantly, which means that the performance of the methodology improves. The computation time reduction is of one third and the efficiency increases $33.33 \%$. It is possible to conclude that a thorough analysis of parameterization in meta-heuristic algorithms has an impact on the efficiency of the algorithm, thus increasing the application field in which these kinds of methodologies can be used.

\section{References}

[1] Abdelaziz, A. Y., E. S. Ali, and SM Abd Elazim. "Optimal sizing and locations of capacitors in radial distribution systems via flower pollination optimization algorithm and power loss index." Engineering Science and Technology, an International Journal 19.1 (2016): 610-618.

[2] Da Silva E. L., Areiza Ortiz J. M., de Oliveira G. C. and Binato S., Transmission network expansion planning under a tabu search approach, IEEE Transactions on Power Systems, Vol. 16, No. 1, pp. 62-68, 2001

[3] Huang S.-J., Application of genetic based fuzzy systems to hydroelectric generation scheduling, IEEE Transactions on Energy Conversion, Vol. 14, No. 3, pp. 724-730, 1999

[4] Huang CM., Huang CJ., Wang ML. A particle swarm optimization to identifying the ARMAX model for shortterm load forecasting. IEEE Transactions on Power Delivery 2005 Vol. 20, N², pp 1126-1133.

[5] Lu Z., Wen Y. and Yang L., An Improved ACO algorithm for service restoration in power distribution systems, Power and Energy Engineering Conference, 2009. APPEEC 2009.Asia-Pacific

[6] Garva, Nitin, and Abhishek Sanghi. "Estimation of Optimal Location and Sizing of DG for Minimization of Loss In Radial Distribution System Using Meta-Heuristic Technique." (2016).

[7] Akorede MF, Hizam H, Aris I, Ab Kadir MZA. Effective method for optimal allocation of distributed generation units in meshed electric power systems. IET Generation, Transmission and Distribution 2011

[8] Mithulananthan N, Oo Than, Van Phu Le. Distributed generator placement in power distribution system using genetic algorithm to reduce losses. Thammasat International Journal of Science and Technology 2004

[9] Borges C. L. T., Manzoni A., Viveros E. C. and Falçao D. M., A parallel genetic algorithm based methodology for network reconfiguration in the presence of dispersed generation, 17 th International Conference
Electricity Distribution (CIRED), Barcelona, Spain, May 2003

[10] Sudha Devi, m. Geethanjali, "Optimal location and sizing determination of Distributed Generation and Transactions on Power Electronics 30.12 (2015): 67916803.

[11] De León-Aldaco, Susana Estefany, Hugo Calleja, and Jesús Aguayo Alquicira. "Metaheuristic optimization methods applied to power converters: A review." IEEE Transactions on Power Electronics 30.12 (2015): 6791-6803 [12] Civanlar S., Grainger J. J., Yin H. and Lee S. S. H., Distribution Feeder Reconfiguration for Loss Reduction, IEEE Transactions on Power Delivery, Vol 3, No. 3, pp. 1217-1223, July 1988

[13] Queiroz LMO, Lyra C. Adaptive hybrid genetic algorithm for technical loss reduction in distribution networks under variable demands. IEEE Transactions on Power Systems 2009

[14] Kashem MA, Jlasmon GB, Mohamed A, Moghavvemi M. Artificial neural network approach to network reconfiguration for loss minimization in distribution networks. Electrical Power and Energy Systems 1998

[15]Jeon Young-Jae, Kim Jae-Chul, Kim Jin-O, Shin JoongRin, Lee Kwang Y. Anefficient simulated annealing algorithm for network reconfiguration in largescale distribution systems. IEEE Transactions on Power Delivery 2002

[16] Jeon Y.-J., Kim J.-C-, Kim J.-O, Shin J.-R, Lee K.-Y, An efficient simulated annealing algorithm for large scale distribution systems, IEEE Transactions on Power Delivery 2002, Vol. 17, No 4, pp. 1070-1078

[17] Zhang D., Fu Z. and Zhang L., An improved TS algorithm for loss-minimum reconfiguration in large-scale distribution systems, Electric Power

[18]Carpaneto E. and Chicco G., Ant-Colony Search-Based Minimum Losses Reconfiguration of Distribution Systems, Proceedings of the IEEE Mediterranean Electrotechnical Conference (MELECON 2004), Dubrovnik, Croatia, Vol. 3, pp. 971-974, May 2004

[19]Su C.-T., Chang C.-F. and Chiou J.-P., Distribution network reconfiguration for loss reduction by ant colony search algorithm, Electric Power Systems Research, Vol. 75, No. 2-3, pp. 190-199, August 2005

[20] Wu Y.-K., Lee C.-Y., Liu L.-C. and Tsai S.-H., Study of Reconfiguration for the Distribution System With Distributed Generators, IEEE Transactions on Power Delivery, Vol. 25, No. 3, pp. 1678-1685, July 2010 [21]O. Abarrategui, I. Zamora, DM. Larruskain "New Metaheuristic Methodology for Loss Reduction through Feeder Reconfiguration"IJEIT Vol 4, Issue11 May 2015 